\title{
Spatial distribution of vector borne disease agents in dogs in Aegean region, Turkey
}

\author{
Distribución espacial de agentes patógenos transmitidos por \\ vectores en perros en la región del Egeo, Turquía
}

Kerem Ural, Ph.D, Mehmet Gultekin, Ph.D, Abidin Atasoy, Ph.D, Bulent Ulutas, Ph.D.

Adnan Menderes University, Faculty of Veterinary, Department of Internal Medicine, Isikli. Merkez Kampüsü Aytepe Mevkii, 09016, Aydin-Turkey. Corresponding: uralkerem@gmail.com

Received: September 2013; Accepted: December 2013.

\begin{abstract}
Objective. Assess the spatial distribution of seroprevalence of infection with or exposure to 4 vectorborne pathogens Ehrlichia canis, Anaplasma phagocytophilum, Borrelia burgdorferi and Dirofilaria immitis, across the coastal states of the Aegean region with special reference to clinical signs and haematological variances related to disease condition. Materials and methods. A convenience sample, targeting blood from at least 10 pet dogs from İzmir, Aydin, Denizli, Mugla and Manisa cities involved was evaluated using a canine point-of-care ELISA kit. Results. Out of 307 dogs tested the overall seroprevalence was highest for $E$. canis $(24.42 \%)$, followed by $E$. canis $+A$. phagocytophilum co-infection (10.42\%), A. phagocytophilum (7.49\%) and D. immitis $(2.28 \%)$. Only 2 cases were seropositive to $B$. burgdorferi albeit 10 dogs were co-infected with more than 2 agents. For both dogs infected with $E$. canis and co-infected with $E$. canis and $A$. phagocytophilum, anemia, thrombocytopenia and leukocytosis, were more commonly detected, whereas thrombocytopenia and leukocytosis were significant finding in dogs infected with $A$. phagocytophilum or $D$. immitis, respectively. Variance analysis showed significant differences for mean RBC, Hb, PCV and PLT values $(p<0.01)$ among control group and other groups. Conclusions. Seropositivity for vector-borne pathogens other than B. burgdorferi, is moderately to widely distributed in dogs residing in the Aegean region in Turkey.
\end{abstract}

Key words: Anaplasma phagocytophilum, Borrelia burgdorferi, Dirofilaria immitis, dogs, Ehrlichia canis, spatial distribution, Turkey (Source: $C A B$ ).

\section{RESUMEN}

Objetivo. Evaluar la distribución espacial de la seroprevalencia de la infección de 4 agentes patógenos de transmisión por vectores Ehrlichia canis, Anaplasma phagocytophilum, Borrelia burgdorferi y Dirofilaria immitis, en los estados costeros de la región del Egeo con especial referencia a los signos clínicos y las variaciones hematológicas relacionadas con la enfermedad. Materiales y métodos. Se tomaron por conveniencia muestras de sangre de al menos 10 perros en las ciudades Izmir, Aydin, Denizli, Mugla y Manisa. Para la evaluación de las muestras se utilizó un kit de ELISA para la detencción de anticuerpos de las enfermedades del estudio. Resultados. De los 307 perros muestreados, la seroprevalencia más alta fue para $E$. canis $(24.42 \%)$, seguido por la coinfección entre $E$. canis $+A$. 
phagocytophilum (10.42\%), A. phagocytophilum (7.49\%) y D. immitis (2.28\%). Sólo 2 casos fueron seropositivos para $B$. burgdorferi aunque 10 perros fueron coinfectados con más de 2 agentes. En ambos perros infectados con $E$. canis y coinfectados con $E$. canis y $A$. phagocytophilum, se detectó comúnmente anemia, trombocitopenia y leucocitosis, mientras que la trombocitopenia y leucocitosis fueron significativos en perros infectados con $A$. phagocytophilum o $D$. immitis, respectivamente. El análisis de varianza mostró diferencias significativas para los promedios de RBC, hemoglobina, hematocrito y valores PLT $(p<0.01)$ entre el grupo control y los otros grupos. Conclusiones. La seropositividad transmitida por vectores patógenos distintos de $B$. burgdorferi, fue moderada y ampliamente distribuida en los perros que residen en la región del Egeo en Turquía.

Palabras clave: Anaplasma phagocytophilum, Borrelia burgdorferi, Distribución espacial, Dirofilaria immitis, Ehrlichia canis, perros, Turquía (Fuente: $C A B$ )

\section{INTRODUCTION}

The canine vector-borne infectious diseases are emerging problems in veterinary medicine, besides the zoonotic potential of the latter causative agents may carry of importance for human health. Tentative diagnosis, therapy applications and prevention of those infections is dependent upon identification of immunopathology of relevant diseases. Despite prospecting advances have been explored in molecular diagnosis and epidemiology of these infections and their vectors, principle pathology knowledge and immunological reflections of the diseases has lagged behind $(1,2)$.

The vector-borne canine disease caused by Dirofilaria immitis, Borrelia burgdorferi, Ehrlichia canis, and Anaplasma phagocytophilum are frequently observed worldwide. Infection with the latter agents may be controlled in partly through vector avoidance and relevant preventive measures, however morbidity and mortality to those diseases continue to occur in dogs. Albeit the usage of acaricides and insecticides may be insufficient for breaking the enzootic transmission cycles. Within the better knowledge of the pathogenesis of the latter agents for animal and human health, there is clearly a need for additional data on the natural occurence and therefore the prevalence of these infections $(1,2)$.

Diagnosis, preventive measurements, and therapy of vector borne diseases among dogs are effective even if the extent of infection in particular geographic areas is taken into consideration (1). The real seroprevalences of selected vector borne pathogens in the present study has, in general, been lacking among dogs in Turkey, as limited seroprevalence for each pathogen was evaluated individually and separetely within limited populations in local areas. Therefore cited references from Turkey is lacking through Science Citation Index journals. In this project according to the authors previous experiences and observations Eagean region of Turkey and relevant provinces were sampled,

\section{INTRODUCCIÓN}

Las enfermedades infecciosas caninas transmitidas por vectores son problemas emergentes en la medicina veterinaria, además del potencial zoonótico de lo anterior los agentes causantes son de importancia para la salud humana. El diagnóstico de presunción, las aplicaciones de terapia y la prevención de estas infecciones es dependiente de la identificación de la inmunopatología de las enfermedades relevantes. A pesar de que se han explorado avances en prospección en el diagnóstico molecular y la epidemiología de estas infecciones y sus vectores, el principio de conocimiento de la patología y las reflexiones inmunológicos de las enfermedades han quedado rezagadas $(1,2)$.

La enfermedad canina transmitida por vectores causada por Dirofilaria immitis, Borrelia burgdorferi, Ehrlichia canis y Anaplasma phagocytophilum, se observan con frecuencia en todo el mundo. La infección con estos últimos agentes puede ser controlada en parte evitando el vector y medidas preventivas pertinentes, sin embargo la morbilidad y mortalidad por estas enfermedades continúa registrándose en perros. Aunque el uso de acaricidas e insecticidas puede ser insuficiente para romper los ciclos de transmisión enzoótica. Según el conocimiento de la patogenia de los anteriores agentes para la salud humana y animal, claramente existe una necesidad de información adicional sobre su ocurrencia natural y por tanto la prevalencia de estas infecciones $(1,2)$.

El diagnóstico, medidas preventivas y la terapia de enfermedades entre perros transmitidas por vectores son efectivas aún si se tiene en cuenta la extensión de la infección en áreas geográficas particulares (1). Las seroprevalencias reales de los patógenos del vector seleccionado en el presente estudio, en general, han sido pocas entre los perros en Turquía, dado que la seroprevalencia limitada para cada patógeno fue evaluada individualmente y por separado dentro de poblaciones limitadas en áreas locales. 
as the latter locations were previously known to have $E$. canis or $D$. immitis positivity, whereas the real question was the seroprevalence rates for $B$. burgdorferi and $A$. phagocytophilum.

Because dogs in the Aegean region of Turkey are not routinely tested by veterinary surgeons in clinic for vector-borne agents, informative data points were needed for this region. The purpose of the present study was to assess the spatial distribution of seroprevalence of infection with or exposure to 4 vector-borne pathogens such as $E$. canis, A. phagocytophilum, B. burgdorferi and $D$. immitis in 5 out of 8 provinces in Aegean region of Turkey, with special reference to clinical signs and hematological variances related to disease condition.

\section{MATERIAL AND METHODS}

Inclusion criteria and sampling. The present study was enrolled among 307 dogs reffered to the Department of Internal Medicine, Faculty of Veterinary, Adnan Menderes University and privately owned small animal clinics in Aegean region (Aydin, Denizli, İzmir, Manisa and Muğla provinces). One hundred fourty nine diseased dogs, referred with histories of at least one of the clinical signs involving anorexia, weight loss, fever, generalized lymphadenopathy, splenomegalia, muscle weakness, bleeding, spontaneous rhinorrhagia, distal limb edema, dyspnoea, and/ or polyarthritis were evaluated using a canine point-of-care ELISA kit for diagnosis of naturally occuring vector borne diseases (Snap 4Dx). Out of 307 dogs, 158 of them (also subjected to a canine point-of-care ELISA kit and found to be negative against all 4 vector borne agents) were also involved and enrolled as control group. The study protocol was approved by the institutional laboratory animals ethics committee of Adnan Menderes University (HADYEK No: B.30.2.A DU.0.00.00.00/050.04/2010/017) and informed written consent was obtained from all of the dogs owners prior to enrollment of the dogs participated in study $(n=307$, at the age of 3 months to 15 years, 157 male, 150 female).

Haematological examination. Blood samples were withdrawn from vena cephalica antebrachii into anticoagulated (EDTA) and nonanticoagulated tubes. Complete blood counts were performed before within Abacus Junior Vet hematology analyzer.

Serological examinations. Serological analyses were performed for differential diagnoses of some selected diseases appeared to have influence on the study and may have the potential effect on the clinical signs. Each sample belonging to diseased and healthy dogs, was tested by use of an ELISA kit (SNAP 4Dx, IDEXX Laboratories,
Por lo tanto, las referencias citadas de Turquía son pocas en la revistas del Science Citation Index. En este proyecto de acuerdo con las experiencias anteriores de los autores se tomaron muestras y observaciones de la región del Egeo en Turquía y las provincias relevantes, ya que en estos lugares se ha conocido previamente resultados positivos para $E$. canis o $D$. immitis, mientras que la pregunta verdadera son las tasas de seroprevalencia para $B$. burgdorferi y A. phagocytophilum.

Ya que los agentes vectoriales de los perros en la región del Egeo de Turquía no son examinados rutinariamente por veterinarios en una clínica, se necesitan de puntos informativos de datos en esta región. El propósito del presente estudio fue evaluar la distribución espacial de la seroprevalencia de la infección o exposición a patógenos transmitidas por 4 vectores $E$. canis, A. phagocytophilum, B. burgdorferi y $D$. immitis en 5 de 8 provincias de la región del Egeo de Turquía, con especial referencia a los signos clínicos y las variaciones hematológicas relacionadas con la condición de la enfermedad.

\section{MATERIALES Y MÉTODOS}

Criterios de inclusión y toma de muestras. El presente estudio vinculó los 307 perros mencionados en el Departamento de Medicina Interna, Facultad de Veterinaria, Universidad Adnan Menderes y pequeñas clínicas veterinarias privadas en la región del Egeo (provincias de Aydin, Denizli, İzmir, Manisa y Muğla). Ciento cuarenta y nueve perros enfermos con un historial de por lo menos uno de los signos clínicos que implican anorexia, pérdida de peso, fiebre, linfadenopatía generalizada, esplenomegalia, debilidad muscular, hemorragias, rinorragia espontánea, edema de extremidad distal, disnea y/o poliartritis fueron evaluados usando un kit de cuidado canino ELISA para el diagnóstico de enfermedades transmitidas por vector que ocurren naturalmente (Snap 4Dx). De 307 perros, 158 de ellos (también sometidos a un kit de cuidado canino ELISA y resultar negativos a todos los 4 agentes transmitidos por vectores) también fueron incluidos y vinculados como grupo de control. El protocolo del estudio fue aprobado por el comité de ética animal de los laboratorios institucionales de la Universidad Adnan Menderes (HADYEK No. B.30.2.ADU.0.00.00.00/050.04/2010/017) y se obtuvo un consentimiento informado por escrito de todos los propietarios de los perros antes de la vinculación de los perros que participaron en el estudio ( $n=307$, con una edad de 3 meses a 15 años, 157 machos, 150 hembras). 
USA) relevant to the protocol listed in the product insert, and as described previously (1). Aforementioned canine point-of-care ELISA kit detects antibodies occuring against to immunodominant proteins of E. canis ( $p 30$ and p30-1), immunodominant protein of $A$. phagocytophilum (msp2) and the $\mathrm{C} 6$ peptide of $B$. burgdorferi, besides detects circulating carbohydrate antigen of $D$. immitis (1). The 4 test results were recorded in an Excel spreadsheet.

Statistical analysis. All the data distribution analysis for normality assumptions (ShapiroWilk test) and homogeneity of variance (Levene test) were evaluated. Kruskal-Wallis test was used for the data variance is not homogeneous or do not dissolve normally. Mann-Whitney $U$ test with Bonferroni correction was used for Post hoc multiple comparisons and $p<0.05$ was considered significant. Results were given as mean \pm standard error.

Clinical signs obtained based on presence/ absence such as nasal discharge, loss of appetite, weight loss, tick infestation, muscle weakness, bleeding, findings associated with an increased bleeding tendency (dermal petechiae, epistaxis), polyarthritis, lymphadenopathy, neurological, cardiac, and respiratory disorders was calculated among the groups in comparison with the control by use of chi-square test of independence.

This study was summarized partially from a national project completely funded by Adnan Menderes University, Research Projects Funding Unit with Project number VTF-10004/2011.

\section{RESULTS}

Animal population enrolled. Dogs $(n=137)$ with naturally occurring infections, were diagnosed by Snap $4 \mathrm{Dx}$ test results, of both sexes (63 male, 74 female) were enrolled into 4 different groups. I. group ( $n=$ 75 ) consisted $E$. canis infected dogs (3 months-15 years of age), II. group involved $A$. phagocytophilum $(\mathrm{n}=23)$ (7 months-15 years of age), III. group E. canis $+A$. phagocytophilum co-infected dogs $(n=32)$ (3 months-12 years of age) and IV. group $D$. immitis infected dogs $(n=7)$ (2 to 6 years old). B. burgdorferi-infected $(n=2)$ or co-infected dogs with more than two etiological agents $[E$. canis $+A$. phagocytophilum $+D$. immitis infected dogs $n=4, E$. canis + Babesia canis infected dogs $n=3$, E. canis + A. phagocytophilum + Hepatozoon canis infected dogs $n=3$ ] were numerically insufficient (totally $n=10$ ) for statistical evaluation, therefore left out of inclusion criteria for intragroup comparison. Dogs were identified as negative within Snap 4Dx $(n=158)$ and available physical examination results, were enrolled as healthy control group (Table 1).
Examen hematológico. Se tomaron muestras de vena cefálica antebraquial en tubos con anticoagulante (EDTA) y sin anticoagulante. Se realizaron conteos sanguíneos completos con el analizador hematológico Abacus Junior Vet.

Exámenes serológicos. Se realizaron análisis serológicos para los diagnósticos diferenciales de algunas enfermedades seleccionadas que parecen tener influencia en el estudio y pueden tener un efecto potencial sobre los signos clínicos. Cada muestra perteneciente a perros enfermos y sanos, fue probada por un kit ELISA (SNAP 4Dx, IDEXX Laboratories, EE.UU.) relevante para el protocolo mencionado en el prospecto del producto y como se describió anteriormente (1). El kit de atención canina ELISA mencionado anteriormente detecta los anticuerpos que se producen contra las proteínas inmunodominantes de $E$. canis (p30 y p30-1), la proteína inmunodominante de $A$. phagocytophilum (msp2) y el péptido C6 de B. burgdorferi, además de detectar el antígeno carbohidrato circulante de D. immitis (1). Los resultados de las 4 pruebas se registraron en una hoja de cálculo Excel.

Análisis estadístico. Se evaluaron todos los análisis de distribución de datos para los supuestos de normalidad (prueba de Shapiro-Wilk) y homogeneidad de la varianza (prueba de Levene). Se utilizó la prueba de Kruskal-Wallis para los datos, la varianza no es homogénea o no se disuelve normalmente. Se utilizó la prueba $U$ de Mann-Whitney con corrección de Bonferroni para comparaciones múltiples Post hoc y un $p<0.05$ fue considerado como significativo. Los resultados fueron mostrados como media \pm error estándar.

Los signos clínicos obtenidos en base en la presencia/ ausencia de cosas tales como la secreción nasal, pérdida de apetito, pérdida de peso, infestación de garrapatas, debilidad muscular, hemorragias, hallazgos asociados con una tendencia creciente de sangrado (petequias cutáneas, epistaxis), poliartritis, linfadenopatía, trastornos neurológicos, cardiacos y respiratorios, fue calculado entre los grupos en comparación con el control mediante el uso de la prueba Chi cuadrado de independencia.

Este estudio fue resumido parcialmente a partir de un proyecto nacional totalmente financiado por la Universidad Adnan Menderes, Unidad de Financiación de Proyectos de Investigación con número de proyecto VTF-10004/2011.

\section{RESULTADOS}

Población animal incluida. Los perros $(n=137)$ con infecciones naturales fueron diagnosticados mediante los resultados de la prueba Snap 4Dx, 
Table 1. Demographic data among groups.

\begin{tabular}{|c|c|c|c|c|c|}
\hline Breed & $\begin{array}{l}\text { Negative } \\
(n=158)\end{array}$ & $\begin{array}{l}\text { E. canis infected } \\
(n=75)\end{array}$ & $\begin{array}{l}\text { A. phagocytophilum } \\
\text { infected }(n=23)\end{array}$ & $\begin{array}{l}\text { E. canis+ A.phagocytophilum } \\
\text { coinfected }(n=32)\end{array}$ & D. immitis \\
\hline Beagle & 1 & - & 1 & - & - \\
\hline Boxer & 5 & - & - & 5 & - \\
\hline Caucasian Shepherd Dog & 1 & - & - & - & - \\
\hline Cavalier King Charles Spaniel & 3 & - & - & - & - \\
\hline Chow Chow & 3 & - & - & - & - \\
\hline Cocker Spaniel & 4 & 6 & - & - & - \\
\hline Collie & 1 & - & - & - & - \\
\hline Crosbred & 65 & 28 & 13 & 17 & 2 \\
\hline Daschund & 1 & - & - & - & - \\
\hline Doberman Pinscher & 2 & 2 & - & - & - \\
\hline English Bulldog & 2 & - & - & - & - \\
\hline French Bulldog & 2 & - & - & - & - \\
\hline German Shepherd Dog & 9 & 4 & 1 & 1 & - \\
\hline Golden Retriever & 14 & 5 & 4 & 5 & 1 \\
\hline Irish Setter & - & - & 2 & 1 & - \\
\hline Jack Russell & 1 & 1 & - & & - \\
\hline Labrador Retriever & 4 & - & - & 1 & - \\
\hline Miniature Pinscher & 1 & - & - & - & - \\
\hline Neapolitan Mastiff & 1 & - & - & - & - \\
\hline Pekingese & 2 & - & - & - & - \\
\hline Pitbull Terrier & - & 1 & - & - & - \\
\hline Pointer & 2 & 1 & - & - & 2 \\
\hline Pug & 1 & - & - & - & 1 \\
\hline Rottweiler & 4 & 6 & 1 & - & - \\
\hline Samojed & - & 1 & - & - & - \\
\hline Schnauzer & - & 1 & - & - & - \\
\hline Siberian Husky & 8 & 1 & - & - & - \\
\hline St. Bernard & 2 & - & - & - & - \\
\hline Terrier & 10 & 10 & 1 & - & 1 \\
\hline Turkish Anatolian Shepherd Dog & 4 & 8 & - & 2 & - \\
\hline West Highland Terrier & 2 & - & - & - & - \\
\hline Yorkshire Terrier & 3 & - & - & - & - \\
\hline \multicolumn{6}{|l|}{ Gender } \\
\hline Female & 81 & 38 & 11 & 19 & 2 \\
\hline Male & 78 & 37 & 12 & 13 & 5 \\
\hline
\end{tabular}

Haematological findings. Among hematological findings evaluated, relevant changes were shown in table 2. For both dogs infected with $E$. canis and co-infected with $A$. phagocytophilum, anemia, thrombocytopenia and leukocytosis, were more commonly detected, whereas thrombocytopenia was a significant finding in dogs infected with A. phagocytophilum. In dogs infected with $D$. immitis there was a remarkable leukocytosis.

Haematological variables obtained were shown in table 3. Variance analysis showed significant differences for mean RBC and PLT values $(p<0.01)$ among control group and other groups. Statistically significant differences were observed for mean values of $\mathrm{Hb}$ and PCV among control group and others, besides between group III and IV. Variance analysis showed significant differences for mean MCV $(p<0.05)$ [among control group and groups II and IV] and MCHC values $(p<0.01)$ [among group II and other groups] (Table 2).

Serological findings. All related data involving spatial distribution of provinces was shown in figures 1 and 2 . de ambos sexos (63 machos y 74 hembras) y fueron divididos en 4 grupos diferentes. El grupo I. $(n=75)$ consistió de perros infectados con $E$. canis (3 meses-15 años de edad), el grupo II. Involucró A. phagocytophilum $(\mathrm{n}=23)$ ( 7 meses-15 años de edad), el grupo III. perros coinfectados con $E$. canis + A. phagocytophilum $(\mathrm{n}=32)$ (3 meses-12 años de edad) y el grupo IV. perros infectados con D. immitis ( $\mathrm{n}=7$ ) (2 a 6 años). infectados con $B$. burgdorferi $(n=2)$ o perros coinfectados con dos o más agentes etiológicos [perros infectados con E. canis $+A$. phagocytophilum $+D$. immitis $\mathrm{n}=4$, perros infectados con E. canis + Babesia canis $\mathrm{n}=3$, perros infectados con $E$. canis $+A$. phagocytophilum + Hepatozoon canis $n=3$ ] fueron numéricamente insuficientes (en total $n=10$ ) para la evaluación estadística, por lo tanto quedaron por fuera de los criterios de inclusión para la comparación entre grupos. Los perros fueron identificados como negativos dentro de Snap 4Dx $(n=158)$ y los resultados de la examinación física disponible, fueron incluidos en el grupo sano de control (Tabla 1). 
Table 2. haematological abnormalities of dogs infected with E. canis/A. phagocytophilum and D. immitis

\begin{tabular}{|c|c|c|c|c|c|}
\hline Haematological findings & $\begin{array}{c}\text { Reference } \\
\text { range }\end{array}$ & $\begin{array}{c}\text { E. canis } \\
(\mathrm{n} / \%)\end{array}$ & $\begin{array}{c}\text { E. canis+ A. } \\
\text { phagocytophilum } \\
(\mathrm{n} / \%)\end{array}$ & $\begin{array}{c}\text { A. phagocytophilum } \\
(\mathrm{n} / \%)\end{array}$ & $\begin{array}{c}\text { D.immitis } \\
(\mathrm{n} / \%)\end{array}$ \\
\hline Leukopenia $\left(\times 10^{9} / \mathrm{I}\right)$ & $6-16$ & $13 / 75(17.3)$ & $4 / 32(12.5)$ & $4 / 23(17.39)$ & $1 / 7(14.28)$ \\
\hline Leukocytosis $\left(\times 10^{9} / \mathrm{I}\right)$ & & $37 / 75(49.3)$ & $24 / 32(75)$ & $6 / 23(26.08)$ & $3 / 7(42.85)$ \\
\hline Anemia (Hct I/I) & $0.40-0.56$ & $47 / 75(62,6)$ & $27 / 32(84.3)$ & $6 / 23(26.08)$ & $2 / 7(28.57)$ \\
\hline Thrombocytopenia $\left(\times 10^{9} / \mathrm{I}\right)$ & $200-500$ & $59 / 75(78.6)$ & $29 / 32(90.6)$ & $11 / 23(47.82)$ & $1 / 7(14.28)$ \\
\hline
\end{tabular}

Table 3. Descriptive statistics relevant to leukocyte and erthrocyte parameters.

\begin{tabular}{|c|c|c|c|c|c|c|}
\hline & Control (n: 158) & $\begin{array}{l}\text { E. canis } \\
(\mathrm{n}: 75)\end{array}$ & A. phagocytophilum (n: 23) & $\begin{array}{c}\text { E. canis }+A . \\
\text { phagocytophilum (n: 32) }\end{array}$ & D. immitis (n:7) & $\boldsymbol{P}$ \\
\hline WBC $\left(\times 10^{9} / I\right)$ & $11.69( \pm 0.29)$ & $13.28( \pm 1.29)$ & $13.97( \pm 1.56)$ & $13.82( \pm 1.33)$ & $17.04( \pm 5.12)$ & \\
\hline $\operatorname{RBC}\left(\times 10^{12} / \mathrm{I}\right)$ & $6.22{ }^{a}( \pm 0.08)$ & $4.64 \mathrm{~b}( \pm 0.18)$ & $5.01^{b}( \pm 0.23)$ & $4.28^{b}( \pm 0.22)$ & $4.48^{b}( \pm 0.63)$ & $*$ \\
\hline $\mathrm{HGB}(\mathrm{g} / \mathrm{dl})$ & $13.74^{\mathrm{a}}( \pm 0.20)$ & $9.99^{b, c, d}( \pm 0,44)$ & $11.01^{\mathrm{c}}( \pm 0.53)$ & $9.21^{d}( \pm 0.50)$ & $9.73^{b, c, d}( \pm 1.30)$ & $*$ \\
\hline HCT (I/I) & $40.46^{\mathrm{a}}( \pm 0.52)$ & $29.46^{b, c, d}( \pm 1.21)$ & $32.64^{\mathrm{c}}( \pm 1.55)$ & $27.43^{\mathrm{d}}( \pm 1.43)$ & $28.54^{b, c, d}( \pm 3.68)$ & $*$ \\
\hline $\mathrm{MCV}(\mathrm{fL})$ & $65.15^{\mathrm{a}}( \pm 0.55)$ & $63.56^{b}( \pm 0.60)$ & $65.26^{\mathrm{a}, \mathrm{b}}( \pm 1.13)$ & $64.38^{b}( \pm 0.74)$ & $64.57^{a, b}( \pm 2.25)$ & $*$ \\
\hline $\mathrm{MCHC}(\mathrm{g} / \mathrm{dl})$ & $34.36^{\mathrm{a}}( \pm 0.16)$ & $33.32^{b}( \pm 0.343)$ & $33.84^{\mathrm{a}}( \pm 0.42)$ & $33.70{ }^{\mathrm{a}}( \pm 0.26)$ & $34.41( \pm 1.11)$ & $*$ \\
\hline PLT $\left(\times 10^{9} / \mathrm{I}\right)$ & $375^{a}( \pm 12)$ & $179 \mathrm{~b}( \pm 23)$ & $207^{b}( \pm 47)$ & $190 \mathrm{~b}( \pm 26)$ & $231^{b}( \pm 39)$ & $*$ \\
\hline
\end{tabular}

$a, b, c$, i Superscripts presented within different letters at the same row indicated statistical significance $(* p \leq 0.01)$. Values were indicated as mean \pm SE mean.

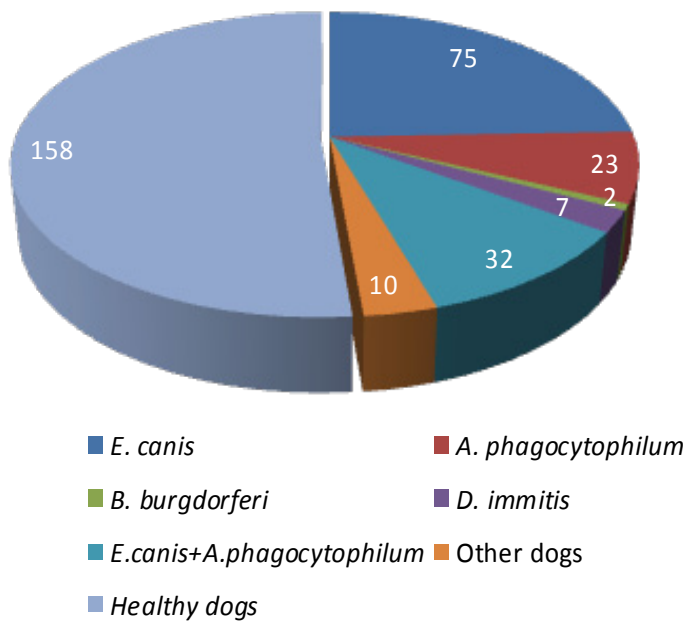

Figure. 1. Snap 4Dx test results relevant to distribution of etiological agents among total study population

Anaplasmosis. An overall prevalence of $7.49 \%$ was detected, besides $10.42 \%$ presented both E. canis and $A$. phagocytophilum antibodies. The highest prevalence of samples with antibodies to A. phagocytophilum were reported from Aydin province $(n=10)$; samples from İzmir also were frequently found to be positive $(n=8)$. None of the dogs tested positive for $A$. phagocytophilum were co-infected with $B$. burgdorferi.

Lyme borreliosis. B. burgdorferi antibodies were detected solely in 2 cases in Kuşadası, Aydın.
Hallazgos hematológicos. Los cambios relevantes entre los hallazgos hematológicos evaluados se muestran en la tabla 2. Para ambos perros infectados con $E$. canis y coinfectados con A. phagocytophilum, anemia, trombocitopenia y leucocitosis, fueron detectados más comúnmente, mientras que la trombocitopenia fue un hallazgo significativo en los perros infectados con $A$. phagocytophilum. En los perros infectados con $D$. immitis se presentó una leucocitosis notable.

Las variables hematológicas obtenidas se muestran en la tabla 3. El análisis de varianza mostró diferencias significativas para los valores promedio de RBC y PLT $(p<0.01)$ entre el grupo de control y los demás grupos. Se observaron diferencias estadísticamente significativas para los valores promedio de hemoglobina y hematocritos entre el grupo de control y los otros grupos, además de entre el grupo III. y el IV. El análisis de varianza mostró diferencias significativas para el valor promedio de MCV $(p<0.05)$ [entre el grupo de control y los grupos II y IV] y los valores promedios de MCHC $(p<0.01)$ [entre el grupo II y los demás grupos] (Tabla 2).

Hallazgos serológicos. En las figuras 1 y 2 se muestran todos los datos relacionados que involucran una distribución espacial de las provincias se.

Anaplasmosis. Se detectó una prevalencia general del $7.49 \%$, además el $10.42 \%$ presentó anticuerpos 

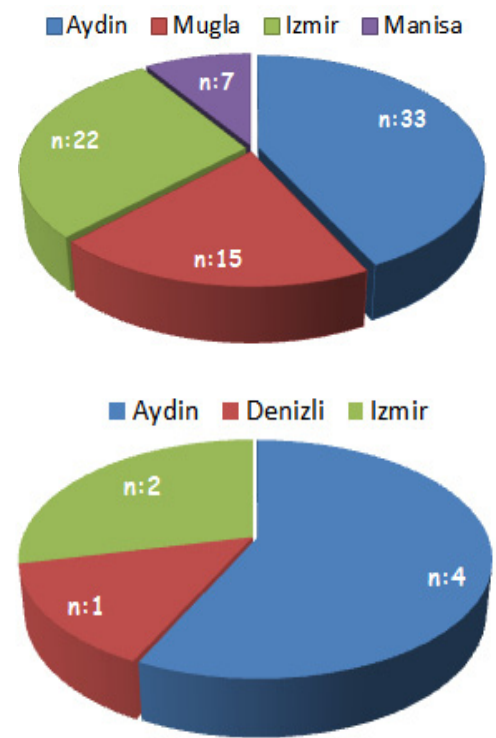
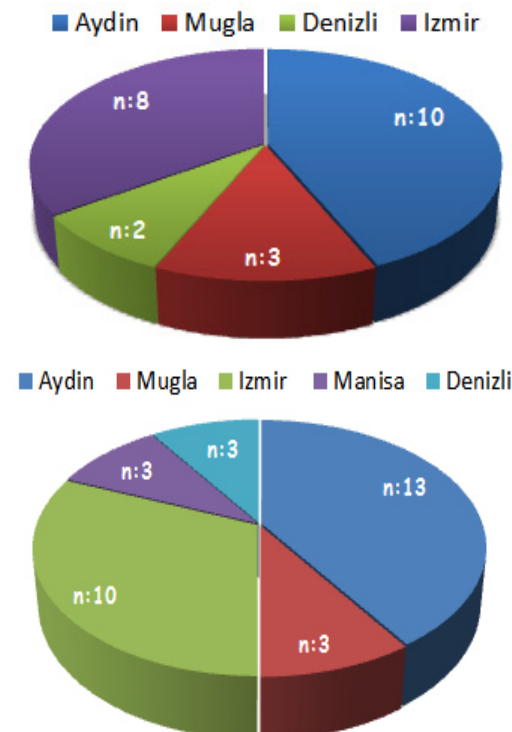

Figure 2. Spatial distribution of vector borne agents according to cities enrolled; a) E.canis, b) A. phagocytophilum, c) D. immitis and d) E.canis+ A. phagocytophilum seropositive cases.

Ehrlichiosis. The overall prevalence for $E$. canis was $24.42 \%$ of 307 dogs. Antibodies to $E$. canis were detected most often in dogs in Aydin $(n=33)$, followed by 22 and 15 cases in Izmir and Mugla provinces, respectively.

Dirofilariosis. The highest percentage of $D$. immitis antigen-positive samples, was obtained from Aydin $(n=4)$ and Izmir $(n=2)$.

Clinical findings. Chi-square test was used for independence controls among groups and clinical findings observed based on presence (1)- or absence (0) such as nasal discharge, anorexia, weight loss, tick infestation, muscle weakness, bleeding tendency (petechiae/ecchymosis, epistaxis), polyarthritis, lymphadenopathy, neurological/cardiological/respiratoric disorders were dependent variables among groups (control, A. phagocytophilum, D. immitis, E. canis $+A$. phagoytophilum and $E$. canis) at the level of $p \leq 0.01$.

Taking into account the probable transmission via the bite of ticks for ehrlichial organisms, active tick infestation was evident among $82.60 \%, 81.25 \%$, and $86.66 \%$ of dogs infected with $A$. phagocytophilum mixed $E$. canis and $A$. phagocytophilum, and E. canis, respectively. Cases were deemed individually, 5 dogs showed corneal and gingival hemorrhage (Figures. 3 a-b), 4 of dogs infected with $E$. canis presented distal limb edema (probably related to deep vein thrombosis) (Figure 3c-d), whereas some of the $A$. phagocytophilum infected dogs showed icterus (Figure $4 a$ ), lameness in association with de E. canis y A. phagocytophilum. La mayor prevalencia de muestras con anticuerpos contra A. phagocytophilum se registró en la provincia de Aydin $(n=10)$; muestras de İzmir también fueron frecuentemente encontradas como positivas $(n=8)$. Ninguno de los perros examinados como positivos para A. phagocytophilum estaban coinfectados con B. burgdorferi.

Borreliosis de Lyme. Se detectaron anticuerpos contra $B$. burgdorferi únicamente en 2 casos en Kusadasi, Aydın.

Ehrlichiosis. La prevalencia general de E. canis fue del $24,42 \%$ entre los 307 perros. Se detectaron anticuerpos contra $E$. canis con más frecuencia en los perros en Aydin $(n=33)$, seguidos por 22 y 15 casos en las provincias de Izmir y Mugla, respectivamente.

Dirofilariosis. El mayor porcentaje de muestras positivas del antígeno de $D$. immitis fueron obtenidas de Aydin $(n=4)$ e Izmir $(n=2)$.

Hallazgos clínicos. Se utilizó la prueba Chicuadrado para controles de independencia entre los grupos y los resultados clínicos observados se basaron en presencia (1)- o ausencia (0) de síntomas como secreción nasal, anorexia, pérdida de peso, infestación de garrapatas, debilidad muscular, tendencia al sangrado (petequias/ equimosis, epistaxis), poliartritis, linfadenopatía, trastornos neurológicos/cardiológicos/respiratorios como variables dependientes entre los grupos (control, A. phagocytophilum, D. immitis, E. Canis + A. phagoytophilum y E. canis) a un nivel de $\mathrm{p} \leq 0.01$. 


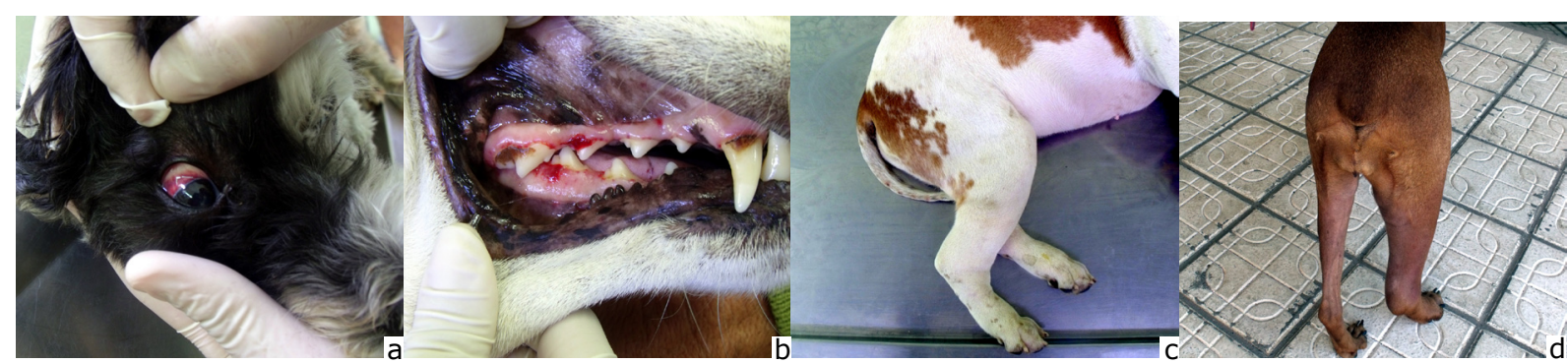

Figure. 3. E. canis seropositive clinical cases showing bleeding tendency a) corneal, b) gingival hemorrhage, c) and d) distal limb oedema due to probable deep vein thrombosis.

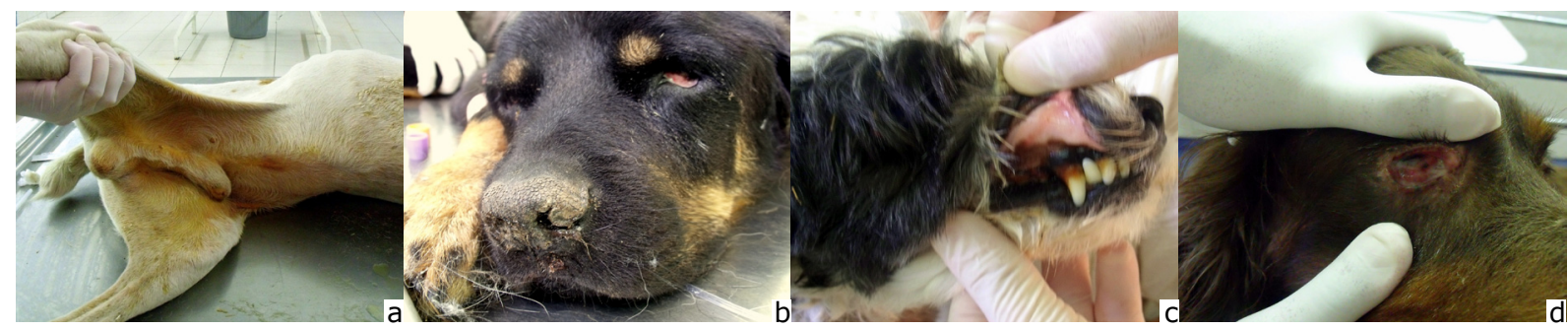

Figure 4. A. phagocytophilum seropositive cases a) generalized icterus, b) a Rottweiler presenting anemia and central nervous signs, c) a Terrier with anemia and d) Irish Setter with ocular hyperemia.

polyarthritis and central nervous system signs (Figure 4b). Dogs infected with $E$. canis or $A$. phagocytophilum presented anemia (Figure 4c), hyperemia (Figure 4d) and bleeding tendency.

\section{DISCUSSION}

Canine granulocytic anaplasmosis has been reported worlwide, possessing parallel geographic distribution of human cases (3). Relatively novel study suggested that $A$. phagocytophilum infections may be seen in humans from different climatic regions among Turkey. In the latter study it was detected that both the seroprevalence of $A$. phagocytophilum and probably co-infections of $A$. phagocytophilum and B. burgdorferi are higher in areas with more suitable habitats for Ixodes ricinus (4). Indeed longitudinal information relevant to $I$. ricinus among European countries are lacking, and it was suggested that higher incidence of the latter agent could have an efficacy on increased seroprevalence in dogs (5).

The detected $A$. phagocytophilum ELISA seroprevalence of $7.49 \%$ in dogs is not comparable, as relevant published data is lacking. The tested dogs of the present study lived most of their life in Aegean region; no seroprevalence data from this region were available among dogs at the time of testing. It should be kept in mind that the vast majority of dogs naturally infected with
Teniendo en cuenta una transmisión probable mediante la picadura de garrapatas para organismos ehrlichiales, una infestación activa de garrapatas fue evidente entre el $82.60 \%, 81.25 \%$ y el $86,66 \%$ de los perros infectados con $A$. phagocytophilum, mezcla de $E$. canis y A. phagocytophilum y E. canis, respectivamente. Los casos fueron considerados individualmente, 5 perros mostraron hemorragia gingival y corneal (Figuras. 3 a-b), 4 perros infectados con $E$. canis presentaron edema de extremidad distal (probablemente relacionado con trombosis venosa profunda) (Figura 3c-d), mientras que algunos de los perros infectados con $A$. phagocytophilum mostraron ictericia (Figura 4a), señales de cojera en asociación con poliartritis y señales del sistema nervioso central (Figura 4b). Los perros infectados con E. Canis o A. phagocytophilum presentaron anemia (Figura 4c), hiperemia (Figura 4 d) y tendencia a sangrar.

\section{DISCUSIÓN}

La anaplasmosis granulocítica canina ha sido reportada en todo el mundo, con una distribución geográfica paralela con los casos humanos (3). Un estudio relativamente novedoso sugirió que las infecciones con $A$. phagocytophilum pueden observarse en seres humanos de diferentes regiones climáticas de Turquía. En este último estudio se detectó que tanto la seroprevalencia de $A$. phagocytophilum y probablemente las coinfecciones de $A$. phagocytophilum y $B$. burgdorferi son mayores en áreas con hábitats más adecuados para Ixodes ricinus (4). En efecto, hace falta información longitudinal relevante para $I$. ricinus entre los países 
A. phagocytophilum may remain clinically healthy. This may be briefly explained with the frequent distribution of anaplasmosis in endemic locations even in the absence of a clinical illness $(6,7)$. Furthermore $A$. phagocytophilum infection may be selflimiting in dogs (3). As positive titers might be indicating previous exposure, the implications for the discovery of seropositive dogs does not involve active infection all the time. Risk factors for $A$. phagocytophilum infected dogs involved season of the year, mostly between April and July $(3,8)$. Although the data was not shown in detail, in our study in agreement with the latter authors, the vast majority of the dogs infected with A. phagocytophilum and/or E. canis were detected in warmer months (between May and July in Aegean region)

In a relatively recent study determining the seroprevalence of anti-B. burgdorferi antibodies in a population of Turkish dogs by use of both enzyme-linked protein A/G assay and novel flow-cytometry-based borreliacidal antibody test, 23.2 and $27.75 \%$ seropositivities, respectively, were detected (9). In another study performed in Aydın and İzmir provinces, in Turkey IgG antibodies were detected by use of ELISA among 49 dogs out of 140 with $35 \%$ seropositivity (10).

Because A. phagocytophilum shares a vector and reservoir host system with $B$. burgdorferi, the geographic distribution of cases of HGA parallels that of Lyme borreliosis (11). Seropositivity for $B$. burgdorferi was similar geographically to that for A. phagocytophilum, especially in a study evaluating the spatial distribution of seroprevalence for 4 vector-borne pathogens, A. phagocytophilum, B. burgdorferi, E. canis and $D$. immitis, across the western coastal states of the United States (1). In the present study among involved dogs a seropositivity of $7.49 \%$ was detected for $A$. phagocytophilum whereas only 2 dogs were seropositive to $B$. burgdorferi. This is surprising given the shared tick vectors (Ixodes spp.) and mammalian reservoirs for both $A$. phagocytophilum and $B$. burgdorferi. The lack of travel history to $A$. phagocytophilum or $B$. burgdorferi seropositive dogs and low seroprevalence rates suggested that disease transmission may be inefficient in Aegean Region, probably due to climate or other environmental conditions, despite the presence of $I$. ricinus. This specificity is of considerable concern in areas with low seroprevalence for $B$. burgdorferi, moreover europeos, y se sugirió que la mayor incidencia del último agente pudo haber sido efectiva en el aumento de la seroprevalencia en perros (5).

La seroprevalencia de $A$. phagocytophilum detectada mediante ELISA del $7.49 \%$ en perros no es comparable, ya que carecen de datos relevantes publicados. Los perros examinados en este estudio vivieron la mayoría de su vida en la región del Egeo; no hay datos disponibles de seroprevalencia en esta región entre perros al momento de la prueba. Debe tenerse en cuenta que la gran mayoría de los perros infectados naturalmente con $A$. phagocytophilum puede permanecer clínicamente sanos. Esto se puede explicar brevemente mediante la distribución frecuente de anaplasmosis en lugares endémicos incluso en ausencia de enfermedades clínicas $(6,7)$. Además, la infección de $A$. phagocytophilum puede ser autolimitante en perros (3). Títulos positivos pueden ser indicativos de una exposición previa, las implicaciones para el descubrimiento de perros seropositivos no implica una infección activa todo el tiempo. Los factores de riesgo para los perros infectados con $A$. phagocytophilum involucraron la temporada del año, principalmente entre abril y julio $(3,8)$. A pesar los datos no fueron expuestos en detalle, en nuestro estudio de acuerdo con estos últimos autores, la gran mayoría de los perros infectados con $A$. phagocytophilum y/o E. canis fueron detectados en los meses más cálidos (entre mayo y julio en la región del Egeo).

En un estudio relativamente reciente donde se determina la seroprevalencia de anticuerpos contra $B$. burgdorferi en una población de perros turcos mediante el uso de una prueba $A / G$ de proteínas de enzimas ligadas y una prueba de anticuerpos borreliacidales en base a citometría de flujo, se detectaron 23,2 y $27,75 \%$ seropositividades, respectivamente (9). En otro estudio realizado en las provincias de Aydın y İzmir, en Turquía, se detectaron anticuerpos IgG mediante el uso de ELISA entre 49 de 140 perros con una seropositividad del 35\% (10).

Dado que $A$. phagocytophilum comparte un sistema de anfitrión vector y reservorio con $B$. burgdorferi, la distribución geográfica de los casos de HGA es paralela a la de la borreliosis de Lyme, (11). La seropositividad para $B$. burgdorferi fue similar geográficamente a la de $A$. phagocytophilum, especialmente en un estudio de evaluación de la distribución espacial de la seroprevalencia para 4 patógenos transmitidos por vectores, A. phagocytophilum, B. burgdorferi, E. canis y $D$. immitis, a través de los estados costeros occidentales de los Estados Unidos (1). En el presente estudio se detectó una seropositividad del $7,49 \%$ para A. Phagocytophilum entre los perros involucrados, mientras que sólo 2 perros fueron seropositivos para $B$. burgdorferi. Esto es sorprendente dada los vectores compartidos de garrapatas (Ixodes spp.) y 
unexposed dogs may be misclassified (1).

Obtained data available in the present study indicated areas where Lyme disease does not appear to be present, suggesting that strong emphasis on prevalence researches of other areas may be required for affected dogs, possibly by use of a more specific test, as Western blot (1). Only scarcity and limited information is available in Turkey relevant to sero-epidemiology of $\mathrm{CME}$, in reality the overall prevalence is underestimated. A prevalence rate of $20.8 \%$ was obtained among 284 dogs in 3 different regions in Turkey (12). In the latter study among cities involved Adana (65.3\%) and Izmir (40.6\%) showed highest prevalences. In previous years, frequently clinical CME cases (13), were reported among dogs in Turkey, whereas among one of the first molecular studies $E$. canis antibodies was detected by use of IFA and dot-ELISA techniques, with $67.8 \%$ and $53.3 \%$ seropositivity rates, respectively (14). In later years in another molecular study 3 out of 12 dogs were seropositive to CME (15). In a recent study in Diyarbakir, Snap 3Dx rapid test kit analysis resulted in $4.8 \%$ seropositivity among 82 dogs (16). Among 4 dogs with CME, 3 were female and only 1 dog was male, and 3 of them were at the age of 4 to 7 years (16). In our study most of the dogs with CME were at the age of 3 months to 15 years of age with no age or sex predisposition.

The most commonly detected haematological alterations were anemia, thrombocytopenia and leukocytosis in dogs infected with $E$. canis and co-infected with $A$. phagocytophilum, whereas thrombocytopenia was a significant finding in dogs infected with $A$. phagocytophilum. Interestingly some of the dogs infected with $E$. canis presented distal limb edema (probably related to deep vein thrombosis), similarly to what have been reported elsewhere $(17,18)$. Hematological variables presented significant differences for mean RBC, $\mathrm{Hb}, \mathrm{PCV}$ and PLT values $(p<0.01)$ among control group and other groups. Although not comparable due to the different nature and status of the diseases involved, it may be suggested that the before mentioned hematological variables showed discrepancy in contrast to healthy controls.

The disease agent for which we observed the highest prevalence on a regional level was $E$. canis. Regarding enrolled population $24.42 \%$ of dogs were positive (solely monoinfection with CME) for $E$. canis on regional pattern, los reservorios de mamíferos para $A$. phagocytophilum y $B$. burgdorferi. La falta de antecedentes de viaje para perros seropositivos para $A$. phagocytophilum o $B$. burgdorferi y las bajas tasas de seroprevalencia sugieren que la transmisión de la enfermedad puede ser ineficiente en la región del Egeo, probablemente debido al clima u otras condiciones ambientales, a pesar de la presencia de $I$. ricinus. Esta especificidad es de considerable preocupación en áreas con una baja seroprevalencia para $B$. burgdorferi, además los perros no expuestos pueden ser clasificados erróneamente (1).

Los datos obtenidos disponibles en el presente estudio indican las áreas donde la enfermedad de Lyme no parece estar presente, sugiriendo que es necesario un fuerte énfasis en investigaciones de prevalencia en otras áreas para los perros afectados, posiblemente mediante el uso de una prueba más específica, como Western blot (1). Sólo está disponible poca información en Turquía sobre la sero-epidemiología de $C M E$, en realidad se subestima la prevalencia general. Se obtuvo una tasa de prevalencia del $20.8 \%$ entre 284 perros en 3 regiones diferentes en Turquía (12). En el último estudio las ciudades involucradas de Adana (65.3\%) e Izmir (40.6\%) mostraron las prevalencias más altas. En años anteriores, los casos clínicos de CME (13) fueron reportados con frecuencia entre perros en Turquía, mientras que en uno de los primeros estudios moleculares se detectaron anticuerpos contra $E$. canis por medio del uso de técnicas IFA y dot-ELISA, con tasas de seropositividad del $53.3 \%$ y $67.8 \%$, respectivamente (14). En los últimos años en otro estudio molecular, 3 de 12 perros fueron seropositivos a CME (15). En un reciente estudio en Diyarbakir, los análisis con el kit Snap 3Dx tuvieron como resultado una seropositividad del $4.8 \%$ entre 82 perros (16). Entre 4 perros con CME, 3 eran hembras y sólo 1 perro era un acho y tres de ellos se encontraban entre los 4 a 7 años de edad (16). En nuestro estudio la mayoría de los perros con CME tenían una edad de 3 meses a 15 años con ninguna predisposición de edad o sexo.

Las alteraciones hematológicas más comúnmente detectadas fueron anemia, trombocitopenia y leucocitosis en perros infectados con $E$. canis y coinfectados con $A$. phagocytophilum, mientras que la trombocitopenia fue un hallazgo significativo en los perros infectados con $A$. phagocytophilum. Curiosamente, algunos de los perros infectados con $E$. canis presentaron edema de miembro distal (probablemente relacionado con trombosis venosa profunda), similarmente a lo reportado en otros lugares $(17,18)$. Las variables hematológicas mostraron diferencias significativas para los valores promedio de RBC, hemoglobina, hematocrito y PLT $(p<0.01)$ entre el grupo de control y los demás grupos. Aunque no es comparable debido a la naturaleza y situación diferente de las enfermedades 
with the highest regional rate observed in Aydin (44\%) and İzmir (29.33\%). Taking into account that brown dog tick thrives near territories inhabited by many stray or kennel dogs, including shelters and some multiple-dog facilities in Aegean Region, it should not be unwise to suggest that E. canis is endemic in this area. Further evaluation of specific environments in which seropositive dogs residing would be helpful for interpretation of suggested data and probably for taking control measures and for developing management strategies. Besides, additional information about the distribution of $R$. sanguineus might be useful for evaluating risks of other probable pathogens transmitted by the latter tick, as reported previously (1). The prevalence of CME may be related to the distribution of the vector, $R$. sanguineus, which occurs mainly in tropical and subtropical regions. The responsible agent causing canine granulocytic ehrlichiosis in Europe has been determined by nucleotide sequencing of the $16 S$ rRNA gene to be identical to the agent of human granulocytic ehrlichiosis (17). The vector of this pathogen in Europe is $I$. ricinus and its reservoir -wild and domestic animals-. In accordance, it was reported that ticks of the genera Ixodes, Rhipicephalus and other relevant ones were widespread throughout Anatolia in Turkey (19).

Interestingly among $E$. canis infected dogs $(n=75), 45.82 \%$ were residing in Aydin city and to those of most of them were living in coastal areas (Kusadasi, Didim etc.). According to the history obtained, owned dogs enrolled in the present study did not resided outside the city borders almost their live, therefore the question raised was the real prevalence of the etiological agents, except Aydin city in Eagean region. The high prevalence of CME in the current study may be explained within several factors involving the presence of $E$. canis in ticks from Aegean Region, frequent exposure of pet dogs to $R$. sanguineus, and sufficient transmission of $E$. canis by the latter tick in warmer climates.

Antigenic testing for Dirofilariosis mostly encountered following the last 15 years among Turkish studies, whereas researhes involving solely one type of diagnostic method (microfilaria or antigenic examination) are the vast majority. Turkish studies detected seropositivity within the rates from ( 0 to $46.22 \%)(20-27)$. Taking into account the latter serosurveys, tha vast majority of them involved limited populations and according involucradas, puede sugerirse que las variables hematológicas mencionadas anteriormente mostraron una discrepancia en contraste con los controles sanos.

El agente de la enfermedad para la cual se observó la mayor prevalencia a nivel regional fue $E$. canis. En cuanto a la población incluida, el $24.42 \%$ de los perros fueron positivos (únicamente monoinfección con $\mathrm{CME}$ ) para $E$. canis en un patrón regional, con la mayor tasa regional observada en Aydin (44\%) e İzmir (29.33\%). Teniendo en cuenta que la garrapata marrón del perro prospera cerca de los territorios habitados por muchos perros callejeros o perreras, incluyendo refugios y algunas instalaciones para múltiples perros en la región del Egeo, no sería poco prudente sugerir que el $E$. canis es endémico en esta zona. Una evaluación adicional de entornos específicos en donde residen perros seropositivos que sería de gran ayuda para la interpretación de los datos sugeridos y probablemente para tomar medidas de control y desarrollar estrategias de manejo. Además, información adicional acerca de la distribución de $R$. sanguineus podría ser útil para evaluar los riesgos de otros patógenos que sea probablemente transmitidos por esta garrapata, como se informó previamente (1). La prevalencia de CME puede estar relacionada con la distribución del vector, $R$. sanguineus, que se presenta principalmente en regiones tropicales y subtropicales. Se ha determinado mediante la secuencia nucleotídica del gen 16s rRNA que el agente responsable que causa ehrlichiosis granulocítica canina en Europa es idéntico al agente de ehrlichiosis granulocítica humana (17). El vector de este patógeno en Europa es I. ricinus y su reservorio son los animales salvajes y domésticos. De esta manera, se informó que las garrapatas del género Ixodes, Rhipicephalus y otras relevantes se distribuyeron ampliamente a lo largo de Anatolia en Turquía (19).

Curiosamente, entre los perros infectados con $E$. canis $(n=75)$, el $45.82 \%$ residían en la ciudad de Aydin y la mayoría de ellos vivían en zonas costeras (Kusadasi, Didim etc.). De acuerdo con el historial obtenido, los perros con amo incluidos en el presente estudio no residían por fuera de los límites urbanos casi toda su vida, por lo tanto, la pregunta planteada era la prevalencia real de los agentes etiológicos, excepto en la ciudad de Aydin en la región del Egeo. La alta prevalencia de CME en este estudio puede explicarse por varios factores que implican la presencia de $E$. canis en garrapatas de la región del Egeo, la exposición frecuente de perros mascota a $R$. sanguineus y la suficiente transmisión de $E$. canis por esta última garrapata en climas cálidos.

Se encontraron principalmente pruebas antigénicas para Dirofilariosis en los últimos 15 años entre los estudios realizados en Turquía, mientras que las investigaciones que involucran únicamente un tipo de método de diagnóstico (microfilaria o exámenes 
to the authors' knowledge none of them reported clinical signs related to disease condition. In a prior serosurvey involving 380 stray dogs from Istanbul and Izmir, a seroprevalence rate of $1.52 \%$ was detected in İstanbul whereas no seropositivity was determined among dogs in Izmir (28). In Aydin city, where the present authors residing and working, microfilaremia was detected with a rate of $13.9 \%$ among 158 dogs (25). In the present research solely 7 dogs presented $D$. immitis antigens. This may be partly explained as a reflection of vector availability. The low prevalence of $D$. immitis found in this study confirmed anectodal evidence that prevalence of Dirofilariasis is indeed not very high in Aegean Region, Turkey. Furthermore it has also been well recognized that mosquito control programmes are well adapted in this region, which might have influence on Dirofilariasis seroprevalence.

Positive serological results may be attributable to prior exposure and not necessarily disease condition, indeed they may forward clinicians to consider further clinical and diagnostic evaluation of dogs for underlying subclinical illness probably requiring treatment. Serologic test results also may be helpful for determining areaspecific disease prevalence, with contribution to understanding distribution of disease in both human and animal patients (1). Albeit to the present authors' experience the seroprevalence studies might possess more scientific value in case of invoving expression of clinical signs. The diseases are managed differently, and all may have complications. Antibacterial chemotherapy and preventive methods, involving vaccination against $B$. burgdorferi, are currently available for all 4 diseases (1), examined in the present study. antigénicos) constituyen la basta mayoría. Los estudios en Turquía detectaron seropositividad dentro de las tasas de 0 a 46.22\% (20-27). Teniendo en cuenta las últimas encuestas serológicas, la inmensa mayoría de las mismas involucraban poblaciones limitadas y según el conocimiento de los autores ninguno de ellos reportó signos clínicos relacionados con la condición de la enfermedad. En una encuesta serológica previa que incluía 380 perros callejeros de Estambul e Izmir, se detectó una tasa de seroprevalencia del $1.52 \%$ en Estambul mientras que no se determinó ninguna seropositividad entre los perros en Izmir (28). En la ciudad de Aydin donde los autores del presente residen y trabajan, se detectó microfilaremia con una tasa del $13.9 \%$ entre 158 perros (25). En esta investigación únicamente 7 perros presentaron antígenos de $D$. immitis. Esto puede ser explicado en parte como un reflejo de la disponibilidad del vector. La baja prevalencia de $D$. immitis encontrada en este estudio confirmó la evidencia anecdótica de que la prevalencia de Dirofilariasis en efecto no es muy alta en la región del Egeo, Turquía. Además, también ha sido reconocido que los programas de control de mosquitos se encuentran bien adaptados en esta región, lo que podría tener una influencia en la seroprevalencia de Dirofilariasis.

Los resultados serológicos positivos pueden ser atribuibles a una exposición previa y no necesariamente a la condición de la enfermedad, de hecho pueden enviar personal clínico para considerar una mayor evaluación clínica y diagnóstica de perros para enfermedades subclínicas subyacentes que probablemente requieren tratamiento. Los resultados de las pruebas serológicas también pueden ser útiles para determinar la prevalencia de enfermedades específicas en un área, como un aporte para comprender la distribución de la enfermedad en pacientes humanos y animales (1). Aunque en la experiencia de los autores los estudios de seroprevalencia podrían poseer un valor científico más alto en caso de involucrar la expresión de signos clínicos. Las enfermedades son manejadas de forma diferente y todas pueden conllevar complicaciones. La quimioterapia antibacteriana y métodos preventivos que implican la vacunación contra $B$. burgdorferi, están actualmente disponibles para todas las 4 enfermedades (1) examinadas en el presente estudio.

\section{REFERENCES}

1. Carrade D, Foley J, Sullivan M, Foley CW, Sykes JE. Spatial distribution of seroprevalence for Anaplasma phagocytophilum, Borrelia burgdorferi, Ehrlichia canis, and Dirofilaria immitis in dogs in Washington, Oregon, and California. Vet Clin Pathol 2011; 40(3):293-302.
2. Bowman D, Little SE, Lorentzen L, Shields J, Sullivan MP, Carlin EP. Prevalence and geographic distribution of Dirofilaria immitis, Borrelia burgdorferi, Ehrlichia canis, and Anaplasma phagocytophilum in dogs in the United States: Results of a national clinic-based serologic survey. Vet Parasitol 2009; 160(1-2):138-48. 
3. Carrade DD, Foley JE, Borjesson DL, Sykes JE. Canine granulocytic anaplasmosis: a review. J Vet Int Med 2009; 23:1129-1141.

4. Gunes T, Poyraz O, Atas M, Turgut NH. The seroprevalence of Anaplasma phagocytophilum in humans from two diff erent climatic regions of Turkey and its co-seroprevalence rate with Borrelia burgdorferi. Turk J Med Sci 2011; 41(5):903-908.

5. Kohn B, Silaghi C, Galke D, Arndt G, Pfister K. Infections with Anaplasma phagocytophilum in dogs in Germany. Res Vet Sci 2011; 91:71-76.

6. Beall MJ, Chandrashekar R, Eberts MD, Cyr KE, Diniz PP, Mainville C, Hegarty BC, Crawford JM, Breitschwerdt EB. Serological and molecular prevalence of Borrelia burgdorferi, Anaplasma phagocytophilum, and Ehrlichia species in dogs from Minnesota. Vector Borne Zoonotic Dis 2008; 8(4):455-64.

7. Foley JE, Foley P, Madigan JE. Spatial distribution of seropsitivity to the caustitive agent of granulocytic ehrlichiosis in dogs in California. Am J Vet Res 2001; 62:1599-1605.

8. Poitout FM, Shinozaki JK, Stockwell PJ, Holland C], Shukla SK. Genetic variants of Anaplasma phagocytophilum infecting dogs in Western Washington State. J Clin Microbiol 2005; 43(2):796-801.

9. Bhide M, Yilmaz Z, Golcu E, Torun S, Mikula I. Seroprevalence of anti-Borrelia burgdorferi antibodies in dogs and horses in Turkey. Ann Agric Environ Med 2008; 15(1):85-90.

10. Uslu O. Köpeklerde Lyme hastalığının araştırılması. [Master Thesis]. Aydın, Turkey: University of Adnan Menderes, Department of Intenal Medicine; 2008.

11. Daniels TJ, Boccia TM, Varde S, Marcus J, Le JH, Bucher DJ et al. Geographic risk for Lyme disease and human granulocytic ehrlichiosis in Southern New York State. Appl Environ Microbiol 1998; 64:4663-4669.

12. Batmaz H, Nevo E, Waner T, Sentürk S, Yilmaz Z, Harrus S. Seroprevalence of Ehrlichia canis antibodies among dogs in Turkey. Vet Rec 2001; 148(21):665-6.

13. Borku MK, Guzel M, Cıngı CC, Ural K, Karakurum MC. Kronik Erlikiozis'li Bir Köpekte Renal Yetmezlik Olgusu. Yuzuncu Yil Univ Saglik Bilim Derg 2003; 14(2):94-96.

14. Erdeğer J, Sancak A, Ataseven L. Detection of Ehrlichia canis in dogs by IFA Test and DotELISA. Turk J Vet Anim Sci 2003; 27:767-773.

15. Unver A, Huang H, Rikihisa Y. Cytokine Gene Expression by Peripheral Blood Leukocytes in Dogs Experimentally Infected with a New Virulent Strain of Ehrlichia canis. Ann N Y Acad Sci 2006; 1078(1):482-486.

16. Icen $H$, Sekin $S$, Simsek A, Kochan A, Celik OY, Altas MG. Prevalence of Dirofilaria immitis, Ehrlichia canis, Borrelia burgdorferi infection in dogs from Diyarbakir in Turkey. Asıan J Anım Vet Adv 2011; 6(4):371-378.

17. Skotarczak B. Canine Ehrlichiosis. Ann Agric Environ Med 2003; 10:137-141.

18. Bressler C, Himes LC, Moreau RE. Portal vein and aortic thromboses in a Siberian husky with ehrlichiosis and hypothyroidism. J Small Anim Pract 2003; 44(9):408-10.

19. Aydin L, Bakirci S. Geographical distribution of ticks in Turkey. Parasitol Res 2007; 101(Suppl 2):163-166.

20. Agaoglu Z, Akgul Y, Ceylan E, Akkan H. Van yöresi köpeklerinde Dirofilaria immitis'in yaygınlığı. YYU Vet Fak Derg 2000; 11(2):41-43.

21. Balıkcı E, Sevgili M. Elazığ ve çevresindeki köpeklerde Dirofilaria immitis'in seroprevalansı. Fırat Univ Sag Bil Vet Derg 2005; 19(2):103-106.

22. Kose K. Erzincan Yöresindeki Köpeklerde Dirofilaria immitis'in Prevalansı Üzerine Araştırmalar. [Master Thesis]. Van, Turkey: University of Yuzuncu Yıl, Institute of Health Sciences; 2005.

23. Simsek S, Utuk AE, Koroglu E, Rishniw M. Serological and molecular studies on Dirofilaria immitis in dogs from Turkey. J Helminthol 2008; 82(2):181-186.

24. Taşçı GT. Kars Yöresi Köpeklerinde Dirofilariosis'in Yaygınlığı. [Master Thesis]. Kars, Turkey: University of Kafkas, Institute of Health Sciences; 2005.

25. Voyvoda H, Paşa S, Ozensoy Toz S, Ozbel Y, Ertabaklar H. Aydın'ın bazı ilçe ve köyleri ile İzmir'in Selçuk ilçesindeki köpeklerde Leishmaniosis ve Diroflariosis'in prevalansı. Turk J Vet Anim Sci 2004; 28:1105-1111.

26. Yildirim A. Ankara ve Çevresindeki Köpeklerde Filarial Etkenlerin Prevalansı. [PhD Thesis]. Ankara, Turkey: University of Ankara, Institute of Health Sciences; 2003.

27. Yildirim A, Ica A, Atalay O, Duzlu O, Inci A. Prevalance and epidemiological aspects of Dirofilaria immitis in dogs form Kayseri province, Turkey. Res Vet Sci 2007; 82:358-363.

28. Oncel T, Vural G. Seroprevalence of Dirofilaria immitis in stray dogs in Istanbul and Izmir. Turk J Vet Anim Sci 2005; 29:785-789. 